\title{
BMJ Open Epidemiology, health systems and stakeholders in rheumatic heart disease in Africa: a systematic review protocol
}

Annesinah Hlengiwe Moloi, ${ }^{1}$ David Watkins, ${ }^{1,2}$ Mark E Engel, ${ }^{1}$ Sumaya Mall,, 3 Liesı Zühlke ${ }^{1,5}$

To cite: Moloi AH, Watkins D, Engel ME, et al.

Epidemiology, health systems and stakeholders in

rheumatic heart disease in

Africa: a systematic review protocol. BMJ Open 2016;6 e011266. doi:10.1136/ bmjopen-2016-011266

- Prepublication history and additional material is available. To view please visit the journal (http://dx.doi.org/ 10.1136/bmjopen-2016011266).

Received 25 January 2016 Revised 31 March 2016 Accepted 28 April 2016

CrossMark

For numbered affiliations see end of article.

Correspondence to

Dr Liesl Zühlke;

liesl.zuhlke@uct.ac.za

\section{ABSTRACT}

Introduction: Rheumatic heart disease (RHD) is a chronic disease affecting the heart valves, secondary to group A streptococcal infection (GAS) and subsequent acute rheumatic fever (ARF). However, RHD cure and preventative measures are inextricably linked with socioeconomic development, as the disease mainly affects children and young adults living in poverty. In order to address RHD, public health officials and health policymakers require up-to-date knowledge on the epidemiology of GAS, ARF and RHD, as well as the existing enablers and gaps in delivery of evidencebased care for these conditions. We propose to conduct a systematic review to assess the literature comprehensively, synthesising all existing quantitative and qualitative data relating to RHD in Africa.

Methods and analysis: We plan to conduct a comprehensive literature search using a number of databases and reference lists of relevant articles published from January 1995 to December 2015. Two evaluators will independently review and extract data from each article. Additionally, we will assess overall study quality and risk of bias, using the Newcastle-Ottawa Scale and the Critical Appraisal Skills Programme criteria for quantitative and qualitative studies, respectively. We will meta-analyse estimates of prevalence, incidence, case fatality and mortality for each of the conditions separately for each country. Qualitative meta-analysis will be conducted for facilitators and barriers in RHD health access. Lastly, we will create a list of key stakeholders. This protocol is registered in the PROSPERO International Prospective Register of systematic reviews, registration number CRD42016032852.

Ethics and dissemination: The information provided by this review will inform and assist relevant stakeholders in identifying key areas of intervention, and designing and implementing evidence-based programmes and policies at the local and regional level. With slight modifications (ie, to the country terms in the search strategy), this protocol can be used as part of a needs assessment in any endemic country.

\section{INTRODUCTION}

\section{Background}

Rheumatic heart disease (RHD) is a chronic inflammatory disease of the heart valves that

\section{Strengths and limitations of this study}

- The review implements a wide range of methods and data to comprehensively and accurately describe rheumatic heart disease (RHD) in Africa, taking into consideration the epidemiology, health systems constraints and stakeholders involved.

- The data will be systematically and rigorously assessed using the most recently published standard quality assessment tools for different study designs. The data ensuing from this review will ultimately inform healthcare policy around RHD in Africa.

- By casting a broad net, we will need to manage a wide variety of literature from different disciplines. We anticipate that reports from nongovernmental organisations and ministry of health documents will be important sources of information for public health planning, so that a broader more inclusive approach can be used for these data without the need for stringent criteria, for example, for systematic reviews of clinical trials.

- There will be significant heterogeneity in the case definitions, which may limit our ability to draw conclusions from the data, particularly objective conclusions.

- The methods for systematic reviews of qualitative research are less well developed, so our analytic approach will be focused on 'broad strokes' and common themes rather than nuanced ethnographic analysis.

usually results from recurrent episodes of acute rheumatic fever (ARF). ARF results from untreated Group A streptococcal (GAS) throat infection (pharyngitis). ${ }^{1-3}$ The clinical signs and symptoms of RHD typically present between the ages of 20 and 50 years. ${ }^{1}$ This clinical period is preceded by a long latent period. RHD tends to be more prevalent in women than in men. In many cases, its first manifestation is during pregnancy, when physiological stress on the heart increases, and this increases the risk of poor fetal and 
maternal outcomes. ${ }^{1}$ It is estimated that RHD is responsible for 233 000-294 000 deaths globally each year. ${ }^{4}$

Primary preventative measures focus on treating GAS pharyngitis with benzathine penicillin in order to prevent ARF. ${ }^{1}$ Secondary prevention measures focus on treating individuals with a history of ARF or RHD, using penicillin as prophylaxis against recurrent ARF and progression of RHD. Guidelines for primary and secondary prevention regimes differ between country and region. Individuals with symptomatic RHD may require a variety of medical or surgical interventions for repair, replacement or palliation of damaged valves. ${ }^{1}$ However, the latent period of RHD poses significant barriers to clinical screening and preventative treatment because individuals are asymptomatic; in fact, up to $50 \%$ of individuals with RHD do not recall an episode of ARF in childhood. ${ }^{5}$ Furthermore, many patients first present to care with advanced heart failure or with other complications, such as infective endocarditis or stroke due to atrial fibrillation. ${ }^{1}$

In low and middle-income countries (LMIC), RHD treatment and prevention is further complicated by lack of access to these interventions at different levels of care. Barriers to healthcare access include: limited community awareness, inadequate health literacy, inadequate health-seeking behaviour and lack of available, affordable and acceptable treatment options. ${ }^{6}$ These frequently occur in the context of a public health and policy environment that does not adequately recognise and does not devote resources to RHD. ${ }^{7}$ At the same time, RHD treatment and prevention measures are inextricably linked with economic status and social development: the disease was largely eradicated during the latter 20th century, in high-income countries. ${ }^{8}$ On the contrary, in LMICs, and especially in African nations, RHD remains the most common cause of acquired heart disease, mostly affecting children and young adults living in poverty. ${ }^{4}$

In order to address RHD, public health officials and health policymakers require information about the epidemiology of GAS, ARF and RHD. They also require specific contextual information about local, regional and national healthcare delivery patterns, and the various stakeholders who would be involved in programmes and policies to prevent and control RHD. This information is particularly important in African nations where RHD continues at a high rate, and resources for adequate prevention and control are especially scarce.

We propose to conduct a systematic review to assess the literature comprehensively, synthesising all existing quantitative and qualitative data concerning the epidemiology of RHD and related health system factors in African nations. We will combine a wide range of methods-further described in the data synthesis section-to paint a complete picture of the current state of RHD disease burden and clinical care. This scientific question by its nature makes our review different from a traditional systematic review. ${ }^{10} 11$

\section{Objectives}

Our systematic review has three objectives: (1) to quantify the burden of RHD, ARF and GAS in African countries; (2) to describe the patient and provider experience with GAS, ARF and RHD care within healthcare systems in these countries; and (3) to identify the types of stakeholders who currently are, or need to be, engaged when designing and implementing RHD programmes in African settings. These objectives are listed in table 1.

\section{METHODS AND ANALYSIS}

This review protocol has been published in the PROSPERO International Prospective Register of systematic reviews (http://www.crd.york.ac.uk/PROSPERO), registration number CRD42016032852. A Preferred Reporting Items for Systematic review and Meta-Analysis Protocols checklist has been completed and included as online supplementary material.

\section{Inclusion criteria}

We will include study articles-published from January 1995 to December 2015-that satisfy the following criteria as appropriate to each of the specified objectives:

\section{Types of studies}

Objective 1: case series, cross sectional studies and cohort studies (retrospective or prospective).

Objective 2: phenomenological and ethnographic (qualitative) studies, including both individual in-depth interviews and focus group discussions.

Objective 3: studies that employ single or mixed methods combining any of the above study designs. We will also consider case reports of RHD programmes or policies in which stakeholders were identified and leveraged.

\section{Types of study subjects}

Objective 1: individuals who reside in geographic regions confined to the African continent.

Objective 2: individuals who utilise or provide RHD healthcare within health systems in African communities.

\begin{tabular}{ll} 
Table 1 & Objectives \\
\hline Objective 1 & $\begin{array}{l}\text { To quantify the burden of RHD, ARF and } \\
\text { GAS in African countries }\end{array}$ \\
Objective 2 & $\begin{array}{l}\text { To describe the patient and provider } \\
\text { experience with GAS, ARF and RHD care } \\
\text { within healthcare systems in these countries }\end{array}$ \\
Objective 3 3 & $\begin{array}{l}\text { To identify the types of stakeholders who } \\
\text { currently are, or need to be, engaged when } \\
\text { designing and implementing RHD } \\
\text { programmes in African settings }\end{array}$ \\
\hline $\begin{array}{l}\text { ARF, acute rheumatic fever; GAS,group A streptococcal infection; } \\
\text { RHD, rheumatic heart disease. }\end{array}$
\end{tabular}


Objective 3: individuals with a vested interest in RHD within African countries (see 'case definitions', below).

\section{Case Definitions}

Objective 1

Group A streptococcal pharyngitis. In a symptomatic participant, GAS can be defined through the use of a clinical predictor rule such as the Walsh pharyngitis criteria, McIsaac decision rule and Centor criteria. ${ }^{12-14}$ Alternatively, a microbiological test such as a throat culture or a rapid antigen test can confirm the presence of GAS in the pharynx of a participant. ${ }^{12}$ To allow for local experiences with GAS and its sequelae, we will note the case definition used in each study and handle any heterogeneity due to different case definitions during the data analysis phase.

Acute rheumatic fever. The diagnosis of the first episode of ARF relies on a constellation of clinical features that follow the Jones criteria (1992) and its subsequent revisions. ${ }^{15}$ We will use these criteria as our ARF case definition in this review, rather than the 2015 revision, as these are still being introduced into standard of care. ${ }^{16}$

Rheumatic heart disease. Prevalence of RHD has in the recent past been defined by screening programmes of subclinical disease in asymptomatic populations, using different criteria. These criteria include those proposed by WHO in 2001, the World Heart Federation in 2012 and authors' criteria adapted for particular screening studies. ${ }^{17-19}$ Hospital-based studies, however, focus on clinical disease in symptomatic populations. As far as possible, we will elucidate the diagnostic methods in either echocardiography screening studies or hospital-based studies.

We will adopt two case definitions for RHD (RHD as diagnosed by a clinician with echocardiographic confirmation or RHD with echocardiographic confirmation only) and explore statistically the impact of different diagnostic criteria on estimates of RHD prevalence.

\section{Objective 2}

We anticipate some variation in case definitions in studies of healthcare delivery. As we are not attempting to estimate population health but rather describe practices around healthcare, we will consider all studies in which participants have been clinically diagnosed with GAS, ARF or RHD. The case definition used in the study will be recorded during the data extraction phase and will be discussed in the analysis if it is deemed to have a substantial impact on interpretation.

\section{Objective 3}

In this study, we will define stakeholders as groups or individuals who have an interest, financial or otherwise, in the implementation or outcomes of healthcare policies, services, practices, processes and/or decisions. ${ }^{20}$ We will consider any studies that clearly define stakeholders involved in any aspect of GAS, ARF and RHD.
Types of outcome measures

\section{Objective 1}

We will seek estimates of one or more of the following epidemiological parameters:

GAS: incidence of GAS pharyngitis and prevalence of GAS among cases of sore throat.

ARF: incidence of ARF and case-fatality rate from ARF.

RHD: incidence of RHD, prevalence of RHD, excess mortality from RHD, case-fatality rate from RHD and rates of non-fatal outcomes such as heart failure, stroke, infective endocarditis, atrial fibrillation and cardiac surgery and its complications.

\section{Objective 2}

We will seek descriptions of RHD healthcare utilisation patterns, specifically barriers and facilitators that may occur along a care pathway. Table 2 presents a conceptual model of the potential barriers and facilitators along this continuum incorporating 'supply' (providers and systems) and 'demand' (patients).

\section{Objective 3}

We will identify and characterise stakeholders and determine inter-relationships as described in the included studies. In the event that we do not find any studies of stakeholders per se, we will utilise the authors and affiliations of the studies from objective 1 and 2 to record information on the various RHD stakeholders identified by the authors of the included studies.

\section{Exclusion criteria}

Opinion, case reports and narrative reviews will be excluded. Systematic reviews will be retained temporarily to manually search reference lists, but ultimately excluded. Publications lacking primary data and/or adequate descriptions of methods will be excluded. Furthermore, if any study has been published in more than one journal/conference, we will exclude all but the most complete and recent version of the data set in question. All unpublished studies must have ethics approval to be considered.

Importantly, we will exclude studies published prior to 1995. The rationale for this date restriction is that we are seeking contemporary information on RHD, to inform

\begin{tabular}{|c|c|c|c|}
\hline & Patients & $\begin{array}{l}\text { Health } \\
\text { providers }\end{array}$ & $\begin{array}{l}\text { Health } \\
\text { Systems }\end{array}$ \\
\hline $\begin{array}{l}\text { Initial decision to seek } \\
\text { care }\end{array}$ & $\checkmark$ & $\checkmark$ & $\checkmark$ \\
\hline $\begin{array}{l}\text { Factors influencing } \\
\text { diagnosis }\end{array}$ & $\checkmark$ & $\checkmark$ & $\checkmark$ \\
\hline $\begin{array}{l}\text { Factors influencing } \\
\text { treatment and/or referral }\end{array}$ & $\checkmark$ & $\checkmark$ & $\checkmark$ \\
\hline $\begin{array}{l}\text { Factors influencing } \\
\text { adherence and retention } \\
\text { in long-term care }\end{array}$ & $\checkmark$ & $\checkmark$ & $\checkmark$ \\
\hline
\end{tabular}


programme development. We also note that echocardiography has only been widely used for diagnosis and screening of RHD in the past two decades.

We anticipate that reports from non-governmental organisations and ministry of health documents will be important sources of information for public health planning, so that a broader and more inclusive approach will be used to review these data.

\section{Search strategy and process}

We have developed a comprehensive search strategy that incorporates a combination of free term text items and Medical Subject Headings (MESH) terms. In addition, to maximise the likelihood of finding articles from Africa, we have applied an Africa-specific search filter described previously by Pienaar $e t a l .^{21}$ Their search strategy was appropriately modified to suit the vocabulary of individual database(s). The complete search strategy, incorporating terms relevant to all three objectives, is shown in tables 3 and 4 for PUBMED and EMBASE, respectively.

Two reviewers will independently screen titles, abstracts and full-text articles. Our primary searches will be conducted on PUBMED, EMBASE and the Worldcat database of dissertations. We will search conference proceedings of the Lancefield Symposia, the Pan African Society of Cardiology, the World Congress of Cardiology and the World Congress of Paediatric Cardiology and Cardiac Surgery, for additional publications and abstracts. We will also search Google Scholar. We will contact relevant departments in the Ministries of Health to obtain death notification data for mortality estimates. Also, experts and in-country university librarians in the field will be consulted to identify additional information such as relevant NGO reports and other unpublished work. Finally, the reference lists of all potentially identified papers will be manually searched for possible studies that were missed by our initial search. Search results from individual databases, reference searches and unpublished articles, will be imported and managed with Covidence (2013 Covidence).

\section{DATA EXTRACTION AND ANALYSIS}

\section{Data extraction and management}

Two reviewers will independently appraise and extract data from each full-text article. Contradictions will be resolved through consensus. A third reviewer will act as an arbitrator where necessary. Using a standardised data extraction form, we will extract the following descriptive and outcome data from each study:

- Study identification: authors, year of publication.

- Eligibility criteria: inclusion and exclusion into the review.

- Study characteristics: study design, characteristics of study participants (ie, demographic information), length of participation, country, study setting (rural, urban, peri urban), number enrolled into the study.
- Case definition: see inclusion criteria.

- Details of the outcome measure: (1) GAS, ARF and RHD epidemiology, (2) Barriers and facilitators, (3) Stakeholders.

- Quality assessment: see below.

- Notes: general study comments.

\section{Data synthesis and analysis Objective 1}

We will meta-analyse estimates of prevalence, incidence, case fatality and mortality for each of the conditions. Before any meta-analysis is conducted, heterogeneity will be assessed by (1) visualising the overlap of CIs in forest plots and (2) calculating the $\mathrm{I}^{2}$ statistic, with caution taken in cases where $\mathrm{I}^{2}$ exceeds $50 \% .^{22}$ Inverse varianceweighted random effects models will be used on logittransformed prevalence and case-fatality ratios, and on log-transformed incidence and mortality rates. We will also explore reasons for heterogeneity, using subgroup analyses or random effects meta-regression on the following parameters: study location, year of study and case definition. If the data are limited or cannot be pooled due to substantial heterogeneity, the findings will be presented in narrative form.

\section{Objective 2}

We anticipate that the data relevant to the second objective will be predominately qualitative. Qualitative meta-analysis (meta-synthesis) aims to uncover similarities and differences across individual studies, and thus generate new insights and areas of inquiry, rather than reduce the findings of multiple studies to a series of pooled summary statistics. ${ }^{23}$

Data synthesis will occur in three overlapping stages. First, we will code the findings of individual primary studies according to their meaning and content (ie, barriers and facilitators and their determinants). Second, we will organise the 'codes' into related areas to construct 'descriptive' themes. ${ }^{24}$ These will be listed in a data display matrix based on our conceptual model (table 1). Third, we will develop 'analytical' themes based on our insights, which can then be incorporated into an explanatory model of health-seeking behaviours and their determinants around GAS, ARF and RHD.

We also anticipate that some studies will present numerical estimates of barriers and facilitators, for example, the proportion of patients with RHD not taking secondary prophylaxis due to disease stigma. We will extract these estimates and present them in the data display matrix. If the number of repeated estimates and quality of the data for any barrier or facilitator are sufficient, we will conduct a quantitative meta-analysis, using methods similar to those used in objective 1 .

\section{Objective 3}

We will create a list of the stakeholders, classifying them by organisational affiliation, qualification/expertise, group represented and impact on the deliberative process. The 
Table 3 PubMed search strategy

Subject
\#1 Group A $\beta$-Haemolytic Streptococcal, Acute

Rheumatic Fever, Rheumatic Heart Disease

\section{Service Delivery}

\#3 Stakeholder Analysis

\#4 Country

\section{Search terms}

"Pharyngitis" [Mesh] OR Pharyngitis OR Sore Throat OR Strep OR Group A $\beta$-Hemolytic Streptococcal OR "Streptococcus Pyogenes" [Mesh] OR Group A Streptococcus OR Group A Streptococcal Infection OR "impetigo" [MeSH] OR impetigo OR group a streptococcus skin infection OR Rheumatic fever OR "Rheumatic Fever" [Mesh] OR Rheumatic heart Disease OR "Rheumatic Heart Disease" [Mesh]

Accessibility of health services OR Availability of health services OR Attitudes to Health OR Barrier* OR Delivery of Health Care OR Facilitator* OR Health OR "Health Behavior" [MeSH] OR Health Care OR Health Care Quality, Access, and Evaluation OR "Health Care personnel OR Health Education" [MeSH] OR Patient compliance OR Patient education OR Professional Knowledge OR Obstacle*

Collaboration OR Health Policy OR Mapping OR "Policy" [MeSH] OR Policy OR Stakeholder

africa[tw] OR africa'[tw] OR africa's[tw] OR africa1[tw] OR africa2[tw] OR africaans[tw] OR africacollaborations[tw] OR africae[tw] OR africaeaustralis[tw] OR africahiv[tw] OR africaid[tw] OR africaid's[tw] OR africain[tw] OR africaine[tw] OR africaine's[tw] OR africaines[tw] OR africains[tw] OR africal[tw] OR africam[tw] OR africamum[tw] OR african[tw] OR african'[tw] OR african"[tw] OR african's[tw] OR african1[tw] OR african2[tw] OR africana[tw] OR africanae[tw] OR africanalleles[tw] OR africanamerican[tw] OR africanan[tw] OR africanane[tw] OR africananes[tw] OR africanasian[tw] OR africanastrongylus[tw] OR africancalotropis[tw] OR africander[tw] OR africanders[tw] OR africane[tw] OR africanendemic[tw] OR africanene [tw] OR africanenes[tw] OR africanensis[tw] OR africanenvironment [tw] OR africaner[tw] OR africanes[tw] OR africani[tw] OR africanised [tw] OR africanism[tw] OR africanist[tw] OR africanists[tw] OR africanity[tw] OR africanium[tw] OR africanizada[tw] OR africanization [tw] OR africanization'[tw] OR africanize[tw] OR africanized[tw] OR africanized'[tw] OR africanizing[tw] OR africanjournal[tw] OR africannum[tw] OR africano[tw] OR africanoides[tw] OR africanol[tw] OR africanos[tw] OR africanoside[tw] OR africanpatients[tw] OR africanpiper[tw] OR africans[tw] OR africans'[tw] OR africanton[tw] OR africantrinervitermes[tw] OR africantriol[tw] OR africanum[tw] OR africanum'[tw] OR africanumsp[tw] OR africanumt[tw] OR africanus [tw] OR africanus'[tw] OR africanusgen[tw] OR africanz[tw] OR africare[tw] OR africarice[tw] OR africas[tw] OR africasia[tw] OR africative[tw]) OR Algeria[tw] OR Angola[tw] OR Benin[tw] OR Botswana[tw] OR Burundi[tw] OR Cameroon[tw] OR Chad[tw] OR Comoros[tw] OR Congo[tw] OR Djibouti[tw] OR Egypt[tw] OR Eritrea [tw] OR Ethiopia[tw] OR Gabon[tw] OR Gambia[tw] OR Ghana[tw] OR Guinea[tw] OR Jamahiriya[tw] OR Jamahiriya[tw] OR Kenya[tw] OR Lesotho[tw] OR Liberia[tw] OR Libya[tw] OR Libya[tw] OR Madagascar[tw] OR Malawi[tw] OR Mali[tw] OR Mauritania[tw] OR Mauritius[tw] OR Mayotte[tw] OR Morocco[tw] OR Mozambique[tw] OR Mozambique[tw] OR Namibia[tw] OR Niger[tw] OR Nigeria[tw] OR Principe[tw] OR Reunion[tw] OR Rwanda[tw] OR Senegal[tw] OR Seychelles[tw] OR Somalia[tw] OR Sudan[tw] OR Swaziland[tw] OR Tanzania[tw] OR Togo[tw] OR Tunisia[tw] OR Uganda[tw] OR Zaire [tw] OR Zambia[tw] OR Zimbabwe[tw]

\#1 AND \#4

\#1 AND \#2 AND \#4

\#1 AND \#3 AND \#4

Search

Filters: Publication date from 01011995 to 31122015.

*Wildcard term as per database notation; MeSH, medical subject heading. 
Table 4 EMBASE search strategy

Subject
\#1 Group A $\beta$-Haemolytic Streptococcal, Acute

Rheumatic Fever, Rheumatic Heart Disease

\#2 Service Delivery

\#3 Stakeholder Analysis

\#4 Country

\section{Search terms}

'group a streptococcal infection'/exp OR 'streptococcus group a'/exp OR 'group a streptococcal infection' OR 'streptococcus group a' OR 'pharyngitis'/exp OR 'pharyngitis' Sore throat OR 'impetigo' OR 'impetigo'/exp OR 'group a streptococcus skin infection'/exp OR 'rheumatic fever'/exp OR 'rheumatic fever' OR 'rheumatic heart disease'/exp OR 'rheumatic heart disease'

'Accessibility of health services' OR 'Availability of health services' OR 'Attitudes to Health' OR 'Barrier' OR 'Delivery of Health Care' OR 'Facilitator' OR 'Health' OR Health Behavior OR 'Health Care' OR 'Health Care Quality, Access, and Evaluation' OR 'Health Care personnel' OR Health Education OR 'Patient compliance' OR 'Patient education' OR 'Professional Knowledge' OR 'Obstacle' 'Collaboration' OR 'Health Policy' OR 'Mapping' OR 'Policy' OR 'Stakeholder'

'Africa' OR Africa* OR Algeria OR Angola OR Benin OR Botswana OR 'Burkina Faso' OR Burundi OR Cameroon OR 'Canary Islands' OR 'Cape Verde' OR 'Central African Republic' OR Chad OR Comoros OR Congo OR 'Democratic Republic of Congo' OR Djibouti OR Egypt OR 'Equatorial Guinea' OR Eritrea OR Ethiopia OR Gabon OR Gambia OR Ghana OR Guinea OR 'Guinea Bissau' OR 'Ivory Coast' OR 'Cote d Ivoire' OR Jamahiriya OR Jamahiryia OR Kenya OR Lesotho OR Liberia OR Libya OR Libia OR Madagascar OR Malawi OR Mali OR Mauritania OR Mauritius OR Mayote OR Morocco OR Mozambique OR Mocambique OR Namibia OR Niger OR Nigeria OR Principe OR Reunion OR Rwanda OR 'Sao Tome' OR Senegal OR Seychelles OR 'Sierra Leone' OR Somalia OR 'South Africa' OR 'St Helena' OR Sudan OR Swaziland OR Tanzania OR Togo OR Tunisia OR Uganda OR 'Western Sahara' OR Zaire OR Zambia OR Zimbabwe OR 'Central Africa' OR 'Central African' OR 'West Africa' OR 'West African' OR 'Western Africa' OR 'Western African' OR 'East Africa' OR 'East African' OR 'Eastern Africa' OR 'Eastern African' OR 'North Africa' OR 'North African' OR 'Northern Africa' OR 'Northern African' OR 'South Africa' OR 'South African' OR 'Southern Africa' OR 'Southern African' OR 'sub Saharan Africa' OR 'sub Saharan African' OR 'subSaharan Africa' OR 'subSaharan African' OR 'guinea pig' OR 'guinea pigs' OR 'aspergillus niger' \#1 AND \#4 \#1 AND \#2 AND \#4 \#1 AND \#3 AND \#4
Search

Search

Search findings will be displayed in spoke-and-wheel diagrams, flow charts, or narrative form, depending on the nature of the data.

\section{Missing data}

Missing data for each study will be described and discussed as to the extent to which they could alter the results. Where deemed necessary, the studies' authors will be contacted to request for missing information.

\section{Quality appraisal of included studies}

We will assess the quality of included studies to evaluate for risk of bias related to the reliability of the estimates of the outcome measures of the study. A high-quality study is considered to have low risk of bias and a low quality study is associated with higher risk of bias. Our assessment of risk of bias will inform the evaluation of heterogeneity in the pooled analyses, especially for objective 1 .

\section{Objective 1}

We will employ the modified version of the Newcastle-Ottawa Scale (NOS) for quantitative observational studies (see online supplementary appendix 1)..$^{25}$ Briefly, the NOS criteria assess cross-sectional studies, cohort studies and case series reports on various items categorised into three groups: the selection of the study groups, the comparability of the groups and the ascertainment of the outcome of interest. 
To allow for relative comparison between the studies and reduce reviewers' subjectivity, we will add a quantitative scoring system to the risk of bias table to categorise studies as low risk, moderate risk and high risk. ${ }^{25}$

\section{Objective 2}

Evidence from qualitative studies will be evaluated using the 10 criteria adapted from the Critical Appraisal Skills Programme (see online supplementary appendix 2). ${ }^{26}$ This grading tool implements the same broad principals of validity used for quantitative research, however, it puts them in a framework that takes into account the different aims and methods used in qualitative research.

\section{DISSEMINATION}

This protocol will guide a systematic review that generates three important areas of new knowledge. First, it will quantify the contemporary burden of GAS and its sequelae (ARF and RHD) in African settings. Second, it will provide a situational assessment of existing barriers and challenges around delivery of clinical care for GAS, ARF and RHD in African communities. Third, it will identify different stakeholders within the realm of RHD in African countries.

The information provided by this review will inform and assist stakeholders in identifying key areas of intervention, and designing and implementing evidencebased programmes and policies, at the local and regional level. With slight modifications to the search strategy, this protocol can also be used as part of a needs assessment in any endemic country.

A unique strength of our review is that it will draw upon a wide range of research methods and data sources, to comprehensively and accurately describe the opportunities for, and challenges to, RHD prevention and control in Africa. We will systematically and rigorously analyse and assess studies, using up-to-date meta-analysis/meta-synthesis techniques and the most recently published quality assessment tools.

Nevertheless, our review has several limitations. First, by casting a broad net, we will need to manage a wide variety of literature from different disciplines. We anticipate that lower-quality studies will be important sources of information for public health planning, so we will have to relax the usual stringent criteria used, for example, for systematic reviews of clinical trials. Second, we recognise that significant heterogeneity in the case definitions may limit our ability to draw conclusions from the data, particularly for objective 1 . Third, the methods for systematic reviews of qualitative research are less well developed, so our analytic approach will be focused on 'broad strokes' and common themes rather than nuanced ethnographic analysis.

In summary, we anticipate that this review will provide comprehensive data on the epidemiology of GAS, ARF and RHD in African settings, with the additional lens of health systems and stakeholders. These data will inform key areas of intervention for research, practice and policy in endemic RHD countries.

Author affiliations

${ }^{1}$ Department of Medicine, Faculty of Health Sciences, University of Cape

Town, Cape Town, South Africa

${ }^{2}$ Department of Medicine, University of Washington, Seattle, Washington, USA

${ }^{3}$ Department of Psychiatry, Groote Schuur Hospital Cape Town, Cape Town,

South Africa

${ }^{4}$ Centre for Evidence Based Health Care, Faculty of Health Sciences,

Stellenbosch University, Stellenbosch, South Africa

${ }^{5}$ Department of Paediatrics, Red Cross War Memorial Children's Hospital, Cape Town, South Africa

Contributors LZ conceived the review. AHM and DW developed and wrote the first draft, and all the authors edited the subsequent versions of the draft. AHM and DW will perform all the searches, extract data and perform data analysis. SM will carry out only qualitative data extraction and analysis. MEE will oversee the final analysis of the data. All the authors have reviewed and accepted the final version of the protocol, and give their permission for publication.

Funding Funded by Medtronic Philanthropy through support to RHD Action. Competing interests None declared.

Ethics approval This systematic review protocol and subsequent review will draw on publicly available data and therefore does not require formal ethical review.

Provenance and peer review Not commissioned; externally peer reviewed.

Open Access This is an Open Access article distributed in accordance with the Creative Commons Attribution Non Commercial (CC BY-NC 4.0) license, which permits others to distribute, remix, adapt, build upon this work noncommercially, and license their derivative works on different terms, provided the original work is properly cited and the use is non-commercial. See: http:// creativecommons.org/licenses/by-nc/4.0/

\section{REFERENCES}

1. Marijon E, Mirabel M, Celermajer DS, et al. Rheumatic heart disease. Lancet 2012;379:953-64.

2. Carapetis JR, McDonald M, Wilson NJ. Acute rheumatic fever. Lancet 2005;366:155-68.

3. Guilherme L, Kalil J. Rheumatic fever and rheumatic heart disease: cellular mechanisms leading autoimmune reactivity and disease. J Clin Immunol 2010;30:17-23.

4. Carapetis J, Steer A, Mulholland E, et al. The global burden of group a streptococcus disease. Lancet Infect Dis 2005;5:685-94.

5. Veasy LG, Wiedmeier SE, Orsmond GS, et al. Resurgence of acute rheumatic fever in the intermountain area of the United States. N Engl J Med 1987;316:421-7.

6. Zuhlke L, Engel ME, Karthikeyan G, et al. Characteristics, complications, and gaps in evidence-based interventions in rheumatic heart disease: the Global Rheumatic Heart Disease Registry (the REMEDY study). Eur Heart J2015;36:1115-22a.

7. Longenecker C, Lwabi $\mathrm{P}$, Kityo $\mathrm{C}$, et al. Leveraging existing HIV/ AIDS infrastructure for rheumatic heart disease care in Uganda: a collaborative disease surveillance and management program. Glob Heart 2014;9:e55. http://www.embase.com/search/results? subaction=viewrecord\&from $=$ export\&id=L71459886

8. Jones TD, Lecture M, Gordis L. The virtual disappearance of rheumatic fever in the United States: lessons in the rise and fall of disease. T. Duckett Jones memorial lecture. Circulation 1985;72:1155-62. http://circ.ahajournals.org/content/72/6/1155.citation

9. Rheumatic D. Rheumatic heart disease: social and economic dimensions. S Afr Med J 2008;98:780-1.

10. Organization WH. Situation analysis and priority setting. World health organization. 2015 (cited 7 December 2015) p. 1. http://www. who.int/nationalpolicies/processes/priorities/en/

11. Worthington $\mathrm{H} \mathrm{V}$, Esposito $\mathrm{M}$, Nieri $\mathrm{M}$, et al. What is a systematic review? Eur J Oral Implantol 2003;1:174-5.

12. Choby BA. Diagnosis and treatment of streptococcal pharyngitis. Am Fam Physician 2009;79:383-90.

13. Mclsaac WJ, Kellner JD, Aufricht $P$, et al. Empirical validation of guidelines for the management of pharyngitis in children and adults. JAMA 2004;291:1587-95. 
14. Walsh BT, Bookheim WW, Johnson RC, et al. Recognition of streptococcal pharyngitis in adults. Arch Intern Med 1975;135:1493-7.

15. Dajani AS, Ayoub E, Bierman FZ, et al. Special writing group of the committee on rheumatic fever, endocarditis and Kawasaki disease of the council on cardiovascular disease in the young of the American Heart Association: Guidelines for the diagnosis of rheumatic fever-Jones Criteria, 1992 Upda. JAMA 1992;268:2069-73.

16. Gewitz MH, Baltimore RS, Tani LY, et al., American Heart Association Committee on Rheumatic Fever, Endocarditis, and Kawasaki Disease of the Council on Cardiovascular Disease in the Young. Revision of the Jones Criteria for the Diagnosis of Acute Rheumatic Fever in the Era of Doppler Echocardiography A Scientific Statement From the American Heart Association. Circulation 2015;131:1806-18.

17. WHO Study Group on Rheumatic Fever and Rheumatic Heart Disease (2001: Geneva S, Organization WH. Rheumatic fever and rheumatic heart disease: report of a WHO expert consultation, Geneva, 20 October-1 November 2001. Geneva: World Health Organization, 2004.

18. Reményi B, Wilson N, Steer A, et al. World Heart Federation criteria for echocardiographic diagnosis of rheumatic heart disease-an evidence-based guideline. Nat Rev Cardiol 2012;9:297-309.

19. Carapetis J, Parr J, Cherian T. Standardization of epidemiologic protocols for surveillance of post-streptococcal sequaelae: acute rhematic fever, rheumatic heart disease and acute post-streptococal glomerulonephritis. http://www.niaid.nih.gov/ topics/strepThroat/Documents/groupasequelae.pdf (accessed 1 Mar 2016).

20. No AP, Replaces EF, No P, et al. Stakeholder Guide 2014 Stakehold Guid 2014. 2014;15-16.

21. Pienaar E, Grobler L, Busgeeth $\mathrm{K}$, et al. Developing a geographic search filter to identify randomised controlled trials in Africa: finding the optimal balance between sensitivity and precision. Health Info Libr J 2011;28:210-15.

22. Hozo S, Djulbegovic B, Hozo I. Estimating the mean and variance from the median, range, and the size of a sample. BMC Med Res Methodol 2005;5:13.

23. Munro SA, Lewin SA, Smith $\mathrm{HJ}$, et al. Patient adherence to tuberculosis treatment: a systematic Review of qualitative research PLoS Med 2007;4:e238.

24. Thomas J, Harden A. Methods for the thematic synthesis of qualitative research in systematic reviews. BMC Med Res Methodol 2008;10:1-10.

25. Wells GA, Shea B, Connell DO, et al. The Newcastle-Ottawa Scale (NOS) for assessing the quality of nonrandomised studies in metaanalyses. The Ottawa Hospital: Research Institute. 2000 (cited 7 December 2015) p. 1. http://www.ohri.ca/programs/clinical_ epidemiology/oxford.asp

26. Critical Appraisal Skills Programme (CASP). In: Qualitative research checklist. Collaboration for Qualitative Methodologies [Internet]. 2006 (cited 8 December 2015). http://media.wix.com/ugd/dded87 29c5b002d $99342 f 788 c 6$ 